LASERS

\section{Bandwidth boost}

Appl. Phys. Express 8, 022701 (2015)

Introducing an active external cavity has been shown to dramatically increase the bandwidth of a distributed feedback (DFB) laser diode. By adding a 220- $\mu \mathrm{m}$-long feedback section that ends with a high reflectivity mirror to a DFB laser, researchers in Japan experimentally realized a light source with a $3 \mathrm{~dB}$ bandwidth of $40 \mathrm{GHz}$. Furthermore, theoretical simulations predict that this figure could be pushed to as high as $70 \mathrm{GHz}$ following optimization. The increase in bandwidth is due to cross-gain modulation and feedback from the external cavity. The feedback section contains a waveguide, a part of which uses electro-absorption to control the intensity and phase of light travelling through it. The InGaAlAs device emits light in the telecoms window at 1,545 nm. The team at Tohoku University and NTT Photonics Laboratories says that the device could be well-suited as an all-optical high-speed wavelength converter or an optical NOT logic gate for optical signal processing circuitry.

\section{MID-INFRARED}

\section{Femtojoule communication}

Opt. Express 22, A1650-A1658 (2014)

In the past few years several researchers have reported greater than unity efficiency for converting electric power into optical power in LEDs. This $>100 \%$ wall-plug efficiency is explained by a contribution to the pumping by lattice heat that additionally enables net cooling of the diode's semiconductor lattice. Duanni Huang and colleagues from the Massachusetts Institute of Technology in the USA have experimentally created a free-space optical communication channel using such a thermo-electrically pumped infrared LED. This means that the electrical energy required for optical communication can be very low - less than the energy of the photon created. For example, the authors report that they need to input as little as $4.3 \mathrm{meV}$ of electrical energy to create a $520 \mathrm{meV}$ photon (with the deficit coming from thermal energy). Using this device, data has been transmitted at a rate of $3 \mathrm{kbps}$, which equates to a power consumption of only $120 \mathrm{pW}$ of electrical power and an energy per binary digit of only 40 femtojoules. The bit error rate of the transmission was $3 \times 10^{-3}$.

\section{TERAHERTZ PHOTONICS}

\section{Superconducting emitter Appl. Phys. Lett. 105, 202603 (2014)}

Research into generating terahertz $(\mathrm{THz})$ radiation from the superconductor $\mathrm{Bi}_{2} \mathrm{Sr}_{2} \mathrm{CaCu}_{2} \mathrm{O}_{8+\delta}(\mathrm{Bi} 2212)$ has received attention recently, with the aim of creating a new compact $\mathrm{THz}$ light source. However, strong Joule heating associated with the large current flowing through the devices has hampered efforts. Now, T. Kitamura and co-workers from Japan and the USA have developed a stand-alone mesa fabrication procedure that greatly improves the thermal contact of the Bi2212 mesa with a surrounding thermal bath and thus allows the emission of high-power, continuous, broadly tunable $\mathrm{THz}$ radiation from 0.29 to $1.06 \mathrm{THz}$. The Bi2212 rectangular mesa with a $74 \mu \mathrm{m}$ top width, $84 \mu \mathrm{m}$ bottom width, $290 \mu \mathrm{m}$ length and $2 \mu \mathrm{m}$ thickness was fabricated using standard metal masks
HOLOGRAPHY Optical voice recorder
Opt. Lett. 39, 6549-6552 (2014)

An optical voice recorder based on the principle of off-axis digital holography is the latest innovation from Osamu Matoba and co-workers at Kobe University and the Kyoto Institute of Technology in Japan. The system relies on the fact that the pressure of sound waves modulates the refractive index of a medium, which in turn modulates the phase distribution of a light wave. An interferometer equipped with an image sensor can thus detect and store these phase distributions in the form of digital holograms, which contain the information required to reproduce the original sounds. The Japanese team

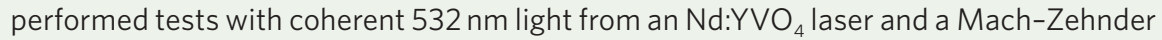
interferometer equipped with a $512 \times 512$ pixel image sensor with a frame rate of $2,000 \mathrm{fps}$. Using this set-up they were able to record and reconstruct sounds from a tuning fork vibrating at $440 \mathrm{~Hz}$ and a human voice at frequencies of up to $1 \mathrm{kHz}$. The highest frequency sound wave that can be reconstructed is half of the frame rate of the camera. As state-of-the-art image sensors are now available with frame rates of $\sim 10^{7} \mathrm{fps}$, in principle ultrasonic waves with frequencies as high as $5 \mathrm{MHz}$ could be recorded using this technique. and Ar ion milling. The top and bottom surfaces were coated with $\mathrm{Ti} / \mathrm{Ag} / \mathrm{Au}$ metal layers and fixed to a sapphire substrate. As the temperature of the device increased from $10 \mathrm{~K}$, the power of the output $\mathrm{THz}$ radiation gradually increased and then suddenly jumped up at a temperature of $54 \mathrm{~K}$, peaked at $56 \mathrm{~K}$ and then decreased sharply above $70 \mathrm{~K}$. The maximum power of the device was estimated to be about $20 \mu \mathrm{W}$. A radiation frequency of $1.06 \mathrm{THz}$ was observed around $10 \mathrm{~K}$, whereas a frequency of $0.29 \mathrm{THz}$ was observed around $70 \mathrm{~K}$. NH

\section{ORBITAL ANGULAR MOMENTUM \\ Broadband generation}

New J. Phys. 16, 123006 (2014)

Optical orbital angular momentum (OAM) has found many applications in imaging, lithography and quantum information. However, most of the existing OAM generators are wavelength dependent and therefore are not compatible with broadband operation. This limitation has now been overcome by Frédéric Bouchard and colleagues at the University of Ottawa in Canada and the University of Rochester in the USA, who have experimentally demonstrated an achromatic spin-toorbital angular momentum convertor with high conversion efficiency. The team used a specially designed system of 'two-Fresnel-rhomb' reflectors (each formed from two glued truncated hollow axicons) to flip the helicity of an incoming circularly polarized beam by inducing a $\pi$ phase shift. Importantly, this induced phase shift is largely independent of the beam's wavelength, thus allowing broadband operation. The team reports an OAM value of \pm 2 and an average conversion efficiency of $95 \%$ for red, green and blue colours. They say that higher OAM values can be obtained by cascading these devices with appropriate waveplates. The broadband operation of the device makes it attractive for a range of imaging applications from microscopic to astronomical systems - such as optical vortex coronagraph, where a white vortex beam is required.

Written by Oliver Graydon, Noriaki Horiuchi, David Pile and Rachel Won.

\section{Correction}

In the Research Highlight 'Dynamic observation' (Nature Photonics 9, 7; 2015), the name of the author of the linked paper was incorrect and should have read Andrew D. Jameson. This has now been corrected in the online versions after print 19 January 2015. 